\title{
Successful Outcome of Elderly Patients with Advanced Malignant Melanoma by Standardized Allergen-removed Rhus verniciflua Stokes Extract and Bojungikki-tang: Two Cases
}

\author{
Hyunsik Jung, Sanghun Lee \\ Department of Medical Consilience, Graduate School, Dankook University \\ 152 Jukjeon-ro, Suji-gu, Yongin-si, Gyeonggi-do, 448-701, South Korea
}

\begin{abstract}
Objectives: Advanced malignant melanoma (MM) has a poor prognosis, with an expected 2-year survival rate of 10 to $20 \%$. It has long been recognized as an immunogenic tumor, and is worse for elderly patients. Many studies have suggested that herbal treatments improve immune functions, but few clinical studies have reported on this topic. Patients and History: We present two cases of female patients (72 and 77 years old, respectively) with advanced MM. The 72-year-old female patient was, at first, diagnosed with MM with multiple bone metastases. She received resection of the primary lesion, but refused further chemotherapy. The 77-year-old female patient was diagnosed with cutaneous MM of the left heel, with suspicion of sentinel node lymphadenopathy; however, she also refused any conventional treatment due to old age.

Course of Therapy and Results: Both patients were exclusively treated with standardized allergen-removed Rhus verniciflua stokes (aRVS) extract combined with Bojungikki-tang (BT, Bu-Zhong-Yi-Qi-Tang in Chinese or Hochu-ekki-to in Japanese). Both patients are still alive and doing well (Feb. 2014), demonstrating that the 72-year-old patient has lived for 27 months and the 77-year-old patient has lived for 31 months without disease progression since the aRVS and BT administration.

Conclusion: We suggest that the combination of aRVS extract and BT could be a candidate for overcoming the cancer's immunoediting process especially for elderly MM patients intolerant of conventional treatment.
\end{abstract}

$\overline{\text { Key Words }}$ : Malignant melanoma, Aged, Rhus verniciflua Stokes, Bojungikki-Tang

\section{Introduction}

Malignant melanoma (MM) is a highly aggressive skin cancer arising from the abnormal proliferation of melanocytes in the basal layer of the epidermis. ${ }^{1)}$ Its invasiveness, metastasis and resistance to conventional cancer therapies, including irradiation and chemotherapy, render its prognosis very poor. ${ }^{2)}$ In fact, the survival rate for patients with metastatic MM is low, with an expected 2-year survival rate of only 10 to $20 \%$. In particular, MM patients over the age of 70 are likely to be characterized by a higher primary TNM stage, more comorbidities and poor performance status. ${ }^{3)}$ Moreover, the aged immune system results in weaknesses of anti-melanoma immunity. ${ }^{4)}$ Therefore, the development of innovative effective treatment is particularly necessary for MM in elderly patients.

Several herbs have been reported to show cytotoxic effects against the human melanoma cell

\footnotetext{
- Received : 22 July 2014

- Revised : 24 October 2014

- Accepted : 24 October 2014

- Correspondence to: Sang-Hun Lee

Department of Medical Consilience, Graduate School, Dankook University

152 Jukjeon-ro, Suji-gu, Yongin-si, Gyeonggi-do, 448-701, South Korea

Tel : +82-31-260-5700, Fax : +82-31-260-5709, E-mail : integrative@korea.com
} 
line as well as improve tumor microenvironments by inhibiting the angiogenesis and modulate immune systems. $^{5-7)}$ Bojungikki-tang (BT, Bu-Zhong-Yi-QiTang in Chinese or Hochu-ekki-to in Japanese) not only have been well known as a typical decoctions improving the general condition by controlling the digestive system, but have also been proven as immunomodulators promoting T-lymphocyte proliferation via experimental studies. ${ }^{8-11)}$ The allergen-removed Rhus verniciflua stokes (aRVS) has been suggested as an antitumor agent by some preclinical and clinical reports. $^{12-14)}$

We presented two cases of MM elderly patients who refused conventional therapies due to their old age and poor performance. With only aRVS and BT treatments, they are still alive beyond their life expectancies, with much improved condition.

\section{Patients and Method}

\section{Patient 1}

A 72-year-old Korean female patient was diagnosed with MM with skull and multiple spine metastases on October 9, 2011 (Fig. 1), having a mass of $1.3 \mathrm{~cm}$ in diameter in the right nasal cavity presenting epistaxis. The $1.6 \mathrm{~cm}$ mass in the nasal cavity was resected with the residual margin involved on October 28, 2011. Chemotherapy was recommended by the physician, but the patient refused due to her old age as well as concerns about quality of life during chemotherapy.

\section{Patient 2}

The other patient, a 77-year-old Korean female, was diagnosed with MM in June 2011, following an abnormal presentation of keratosis and edema on the left heel. Further evaluation, including lymphoscintigraphy, revealed suspicious lymphadenopathy in the left inguinal area. She had been continuously taking hypoglycemic medication for 10 years. Following the primary mass resection, chemotherapy and radiotherapy were recommended; however, her family strongly refused the conventional treatments due to her old age and anxiety about her quality of life after such treatments. Today, she is much older than 77 years, as her birthday was postdated.

\section{Treatment courses}

For these reasons, both patients visited us in order to receive alternative therapies. At the time, they complained of severe fatigue with ECOG performance status 2 , and their symptoms could be categorized as a $q i$ deficiency pattern. The treatment plan did not include any conventional therapies, such as chemotherapy and radiation therapy. Only aRVS extracts with BT have been administrated one hour after each meal since November 7, 2011 (patient 1) and July 19, 2011 (patient 2).

\section{Standardized extract of aRVS and Bojungikki-tang treatment}

Urushiol, a mixture of several derivatives of catechol, must be removed from RVS prior to its pharmaceutical use because it causes severe contact dermatitis in sensitive individuals. The standardized extract of allergen-removed RVS (aRVS) was manufactured based on a thorough historical research. The daily oral administration of 1.350 grams of aRVS extract was prescribed. ${ }^{12)}$

A decoction of BT was prepared from a mixture of chopped crude herbs (Astragalus membranaceus (6g), Panax ginseng C.A.Meyer (4g), Atractylodes macrocephala Koidzumi (4g) Glycyrrhiza uralensis (4g), Angelica gigas Nakai (2g), Citrus unshiu Markovich (2g), Cimicifuga heracleifolia (2g), and Bupleurum falcatum L. (2g)), which were extracted in water at $100^{\circ} \mathrm{C}$ for 2 hours. The quality of the herbs was tested according to the Korea Food \& Drug Administration and our hospital's standards. Oral administration of $100 \mathrm{ml}$ BT decoction was prescribed three times a day. 


\section{Results}

The biochemical parameters associated with liver and renal functions were within the normal range, and no significant adverse effects from the herbal treatment have been observed, except for a mild allergic rash on the thigh (patient 1) and mild pruritus on the head and trunk (patient 2). Follow-up bone scans of patient 1 dated on February 18, 2013 showed no interval change compared to the initial bone scans in 2011 (Fig 2). Patient 2 and her family did not want to receive a follow-up evaluation for her disease, including $\mathrm{CT}$ scans. Both of them maintain a good performance status at the present time (Feb. 2014), which demonstrates that they have lived for 27 and 31 months, respectively, since the aRVS and BT administrations.

\section{Discussion}

The BT formulated by Li Dongyuan (also known as Li Gao; 1180-1252 A.D.) during the Jin-Yuan Dynasty has long been used as the best herbal medicine in Eastern Asia, including Korea, China and Japan. It has been prescribed for patients with Qi-deficiency syndrome, which is defined as a severe weakness caused by general fatigue, loss of appetite, indigestion, poor gastrointestinal functions or excessive sweat. ${ }^{11)}$ Elderly patients are likely to be presented with a general decline in the function of the immune system as well as the gastrointestinal system due to the aging process. ${ }^{15,16)}$

BT was clinically proven to be effective for elderly people to restore age-related impairment of the immune function by enhancing the natural killer (NK) activity against K562 target cells and a significant increase in serum IFN- $\gamma$ levels. ${ }^{16)}$ Additionally, preclinical studies have demonstrated that BT exhibits immunopharmacological activities, including the restoration of anti-tumor T-cell response from stress-induced suppression, induction of mitogenic activity to lymphocytes, dendritic cell maturation, augmentation of NK activity, and increased production of IFN- $\gamma^{8,17-19)}$ The polysaccharide fraction of BT also stimulates antigen-induced $\mathrm{T}$ cell proliferation, production of IFN- $\gamma$ and MHC class II expression. ${ }^{10)}$

The anti-melanoma effect was also demonstrated

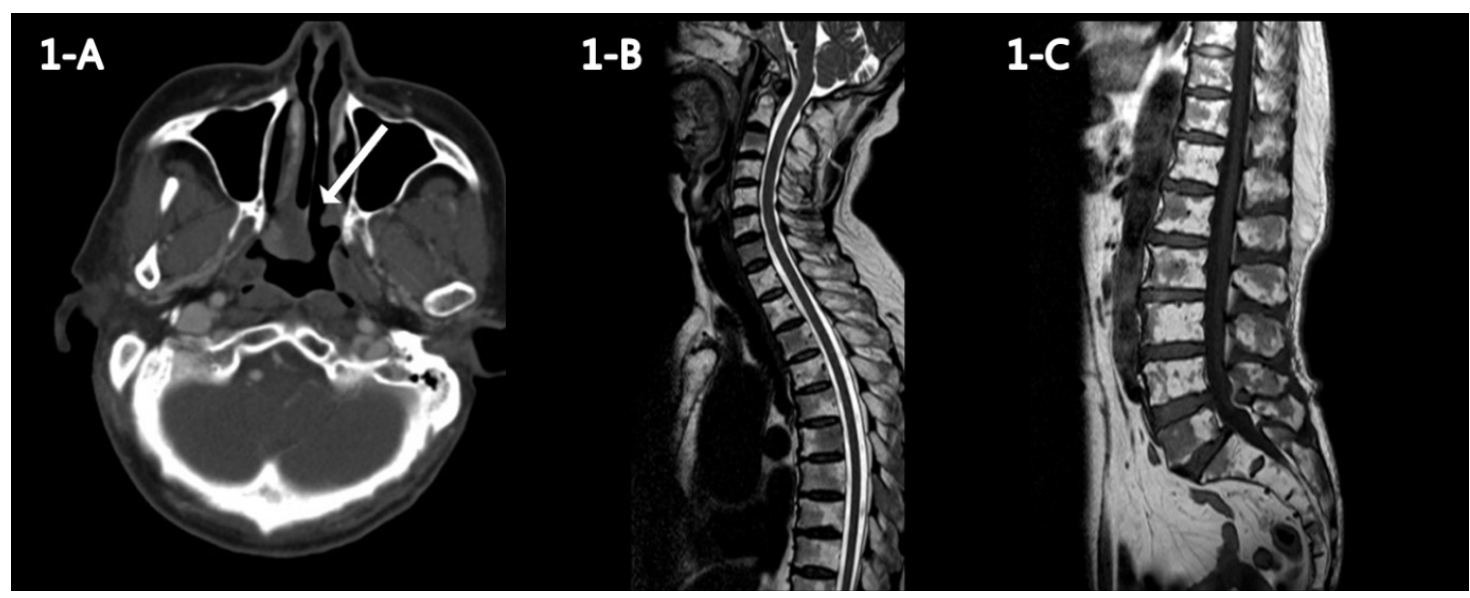

Fig. 1. A neck CT scan dated October 14, 2011 revealed an approximately $2 \mathrm{~cm}$ lobulated mass in the posterior aspect of the right inferior turbinate, abutting to nasal septum (A); A whole spine MRI dated October 27, 2011 showed multiple spine metastases of severe C7, T6, L5 involvement pattern (B and C). 


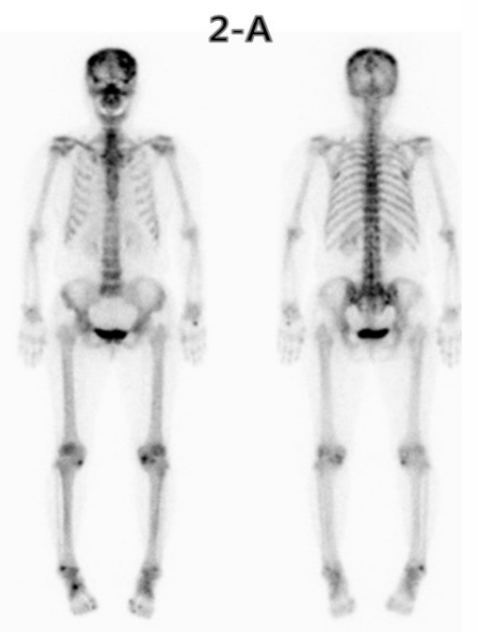

Oct. 2011

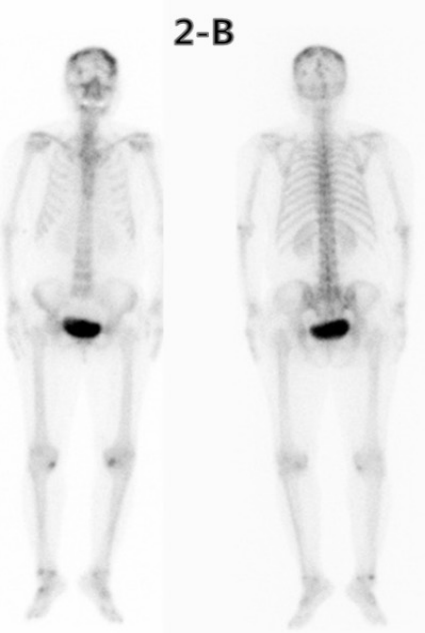

Oct. 2012

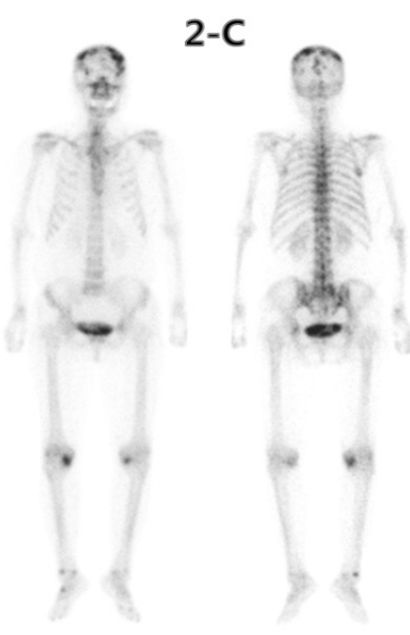

Feb. 2013

Fig. 2. A bone scan dated October 25, 2011 confirmed intensely increased uptake in the nasal bone, bone metastases in skull, sternum, and $T-L-S$ spines $(A)$; The following bone scans after only herbal treatments showed no significant interval change, except for increased osteoblastic lesion of both the knee joint lesion compared with the initial bone scan (B and $\mathrm{C}$ ).

by the Ekongsan decoction composed of Panax ginseng C. A. Meyer (4g), Atractylodes macrocephala Koidzumi (4g) Glycyrrhiza uralensis (4g), Zingiber officinale Roscoe (4g) and Citrus unshiu Markovich (4g), which are quite similar to the BT formula. In a B16 melanoma model using C57BL/6 mouse, it significantly inhibited lung metastasis and increased IL-2 production. ${ }^{6,20,)}$ Its metabolites from human intestinal microflora showed anti-angiogenesis and anti-metastasis in ECV-304 (transformed human endothelial cell) and HT1080 (human fibrosarcoma). The aRVS extract prescribed in the MM cases was also clinically reported to show a complete response in renal cell carcinoma, the immunogenic cancer. ${ }^{21)}$ It was also demonstrated to have anti-angiogenesis and anti-invasion effects. ${ }^{12)}$

Melanoma has long been recognized as an immunogenic tumor based on its response rate to immunotherapy, the incidence of spontaneous regression and the high level of tumor $\mathrm{T}$ cell infiltration. $^{22)}$ Although systemic IFN- $\alpha$ and IL-2 treatments did not show a survival benefit from large randomized trials, the recently developed monoclonal antibodies targeting the inhibitory immune receptors, such as cytotoxic T-lymphocyte-associated antigen-4 (CTLA-4), programmed death 1 (PD1) or its ligand (PD-L1), are very promising. ${ }^{23,24)}$ Nevertheless, the predictable and often dangerous immune-mediated side effects are still challenging.

In advanced MM patients, the expected 2-year survival rate is very low, only about 10 to $20 \%$. Especially, elderly patients like our cases are expected to show poor survival compared to younger patients because of more comorbidities and poor performance status. Therefore, we hope that these MM cases beyond the expected prognosis under only herbal treatments would stimulate further investigation into the effectiveness and tolerability of herbal treatments for elderly patients with $\mathrm{MM}$ in clinical practice, although it should not be generalized for use in all cases. Furthermore, adverse events are very mild compared to conventional 
chemotherapy or immunotherapy agents, and no toxicities were observed for over 2 years. We suggest that the aRVS and BT combination could be a candidate treatment option for improving selective immune function as well as inhibiting MM in elderly patients.

\section{Conflict of interest statement}

We declare that we have no financial or personal relationships with other people or organizations that can inappropriately influence our work.

\section{Acknowledgement}

We would like to thank our patients, who consented to having their cases presented and published.

\section{References}

1. Houghton AN, Polsky D. Focus on melanoma. Cancer Cell. 2002;2(4):275-8.

2. Soengas MS, Lowe SW. Apoptosis and melanoma chemoresistance. Oncogene. 2003;22(20):3138-51.

3. Macdonald JB, Dueck AC, Gray RJ, Wasif N, Swanson DL, Sekulic A, et al., Malignant melanoma in the elderly: different regional disease and poorer prognosis. Journal of Cancer. 2011;2:538-43.

4. Hegde UP, Grant-Kels JM, Metastatic melanoma in the older patient: special considerations. Clinics in Dermatology. 2013;31(3):311-6.

5. Kang $\mathrm{CH}_{\text {, }}$ Shin HK, Shim BS, Kim $\mathrm{SH}$, Choi $\mathrm{SH}$, et al. Experimental Studies on the Anti-angiogenesis and Anti-metastasis Effects of Ekong-san. Journal of Korean Traditional Oncology. 2006;11(1):41-54.
6. Lee SG, Ha JH. Antitumor and immunomodulatory effects of Ikongsan on murine melanoma-induced lung metastasis. Korean Journal of Oriental Medical Pathology. 1997;11(1):21-30.

7. Kwon SJ, Lee JH, Moon KD, Jeong IY, Ahn DU, Lee MK, et al. Induction of apoptosis by isoegomaketone from Perilla frutescens L. in B16 melanoma cells is mediated through ROS generation and mitochondrial-dependent, -independent pathway. Food Chem Toxicol. 2013.

8. Nabeshima S, Murata M, Hamada M, Chong Y, Yamaji K, Hayashi J. Maturation of monocyte -derived dendritic cells by Hochu-ekki-to, a traditional Japanese herbal medicine. Int Immunopharmacol. 2004;4(1):37-45.

9. Cho S, Hong T, Kaneko A, Yoshino G, Sato N, Kikuchi K, et al. Evaluation of immunological effects of hochu-ekki-to (TJ-41) prophylactic administration in mice. Am J Chin Med. 2004; 32(2):235-43.

10. Chae SY, Shin SH, Ha MH, Jo SK, Kim SH, Byun MW, et al. Effect of Bu - Zhong - Yi - Qi - Tang on Proliferation of T Cells. Journal of the Korean Society of Food Science and Nutrition. 2004; 33(7):1085-91.

11. Jeong JS, Ryu BH, Kim JS, Park JW, Choi WC, Yoon SW. Bojungikki-tang for cancer-related fatigue: a pilot randomized clinical trial. Integr Cancer Ther. 2010;9(4):331-8.

12. Choi W, Jung H, Kim K, Lee S, Yoon S, Park $\mathrm{J}$, et al. Rhus verniciflua Stokes against Advanced Cancer: A Perspective from the Korean Integrative Cancer Center. Journal of Biomedicine \& Biotechnology. 2012;2012:874276.

13. Lee S, Kim K, Jung H, Cheon S, Kim S, Eo W, et al. Efficacy and Safety of Standardized 
Allergen-Removed Rhus verniciflua Stokes Extract in Patients with Advanced or Metastatic Pancreatic Cancer: A Korean Single-Center Experience. Oncology. 2011;81(5-6):312-8.

14. Choi W, An S, Kwon E, Eo W, Lee S. Impact of Standardized Allergen-Removed Rhus verniciflua Stokes Extract on Advanced Adenocarcinoma of the Ampulla of Vater: A Case Series. Evidence-based Complementary and Alternative Medicine : eCAM. 2013;2013:203168.

15. Satoh N, Sakai S, Kogure T, Tahara E, Origasa $\mathrm{H}$, Shimada $\mathrm{Y}$, et al. A randomized double blind placebo-controlled clinical trial of Hochuekkito, a traditional herbal medicine, in the treatment of elderly patients with weakness $\mathrm{N}$ of one and responder restricted design. Phytomedicine. 2005;12(8):549-54.

16. Kuroiwa A, Liou S, Yan H, Eshita A, Naitoh S, Nagayama A. Effect of a traditional Japanese herbal medicine, hochu-ekki-to (Bu-Zhong-Yi-Qi Tang), on immunity in elderly persons. Int Immunopharmacol. 2004;4(2):317-24.

17. Yamaoka Y, Kawakita T, Nomoto K. Protective effect of a traditional Japanese medicine Hochu-ekki-to (Chinese name: Bu-zhong-yiqi-tang), on the susceptibility against Listeria monocytogenes in infant mice. Int Immunopharmacol. 2001;1(9-10):1669-77.

18. Utsuyama M, Seidlar H, Kitagawa M, Hirokawa $\mathrm{K}$. Immunological restoration and anti-tumor effect by Japanese herbal medicine in aged mice.
Mechanisms of Ageing and Development. 2001;122(3):341-52.

19. Li T, Tamada K, Abe K, Tada H, Onoe Y, Tatsugami K, et al. The restoration of the antitumor $\mathrm{T}$ cell response from stress-induced suppression using a traditional Chinese herbal medicine Hochu-ekki-to (TJ-41:Bu-Zhong-YiQi-Tang). Immunopharmacology. 1999;43(1): 11-21.

20. Ahn KS, Lee KT, Jang SK, Jeong JC, Noh YS. Experimental Studies on the Immunomodulatory Effects of Ekong-san. Journal of the Institute of Oriental Medicine. 1999;2(-):15-29.

21. Lee SK, Jung HS, Eo WK, Lee SY, Kim SH, Shim BS. Rhus verniciflua Stokes extract as a potential option for treatment of metastatic renal cell carcinoma: report of two cases. Ann Oncol. 2010;21(6):1383-5.

22. Clemente CG, Mihm MC, Jr. Bufalino R, Zurrida S, Collini P, Cascinelli N. Prognostic value of tumor infiltrating lymphocytes in the vertical growth phase of primary cutaneous melanoma. Cancer. 1996;77(7):1303-10.

23. Quezada SA, Peggs KS. Exploiting CTLA-4, PD-1 and PD-L1 to reactivate the host immune response against cancer. British Journal of Cancer. 2013;108(8):1560-5.

24. Maio M. Melanoma as a model tumour for immuno-oncology. Annals of Oncology. 2012; 23:10-4. 